\title{
Quantitative trait loci pyramiding for fruit quality traits in tomato
}

\author{
Adriana Sacco - Antonio Di Matteo • Nadia Lombardi • \\ Nikita Trotta $\cdot$ Biancavaleria Punzo $\cdot$ Angela Mari • \\ Amalia Barone
}

Received: 8 March 2012/ Accepted: 13 June 2012/Published online: 28 June 2012

(C) The Author(s) 2012. This article is published with open access at Springerlink.com

\begin{abstract}
Fruit quality is a major focus for most conventional and innovative tomato breeding strategies, with particular attention being paid to fruit antioxidant compounds. Tomatoes represent a major contribution to dietary nutrition worldwide and a reservoir of diverse antioxidant molecules. In a previous study, we identified two Solanum pennellii introgression lines (IL7-3 and IL12-4) harbouring quantitative trait loci (QTL) that increase the content of ascorbic acid (AsA), phenols and soluble solids (degrees Brix; ${ }^{\circ} \mathrm{Bx}$ ) in tomato fruit. The purpose of the present work was to pyramid into cultivated varieties the selected QTL for enhanced antioxidant and ${ }^{\circ} \mathrm{Bx}$ content. To better understand the genetic architecture of each QTL, the two ILs were crossed to the recurrent parent M82 (ILH7-3 and ILH12-4) and between them
\end{abstract}

A. Sacco - A. Di Matteo - N. Lombardi .

B. Punzo · A. Barone $(\square)$

Department of Soil, Plant, Environmental and Animal

Sciences, University of Naples Federico II, Portici, Italy

e-mail: ambarone@unina.it

N. Trotta

CRA-ORT, Italian Agricultural Research Council,

Research Centre for Vegetable Crops, Pontecagnano, Italy

A. Mari

CNR-ISA, Institute for Food Science, Avellino, Italy

Present Address:

A. Mari

Department of Pharmaceutical and Biomedical Sciences, University of Salerno, Salerno, Italy
(ILH7-3+12-4). F1 hybrids (ILH7-3+12-4) were then selfed up to obtain $\mathrm{F} 3$ progenies in order to stabilize the favourable traits at the homozygous condition. Species-specific molecular markers were identified for each introgressed region and allowed us to select four F2 genotypes carrying both introgressions at the homozygous condition. The F3 double homozygous plants displayed AsA, total phenols and ${ }^{\circ} \mathrm{Bx}$ content significantly higher than M82. Therefore, they may represent suitable genetic material for breeding schemes aiming to increase antioxidant content in tomato fruit.

Keywords Solanum lycopersicum $\cdot$ S. pennellii introgression lines - Molecular markers . Ascorbic acid $\cdot$ Phenols $\cdot$ Soluble solids

Consumers are paying ever-increasing attention to the health and nutritional aspects of horticultural products, such as vitamin content, mineral elements and antioxidants (Causse et al. 2010). Fresh fruit and vegetables have long been regarded as having considerable health benefits, due in particular to their antioxidant content, which can protect the human body against cellular oxidation reactions, decreasing the risk of some common diseases such as cardiovascular events, cancer and other age-related degenerative diseases (Demmig-Adams and Adams 2002). These benefits have stimulated researchers to investigate the antioxidant content of fruit and vegetables, 
and to highlight the molecular mechanisms underlying their synthesis and accumulation in plants.

Tomatoes represent a major contribution to nutrition worldwide and a reservoir of diverse antioxidant molecules, such as ascorbic acid (AsA or vitamin C), vitamin $\mathrm{E}$, carotenoids, flavonoids and phenolic acids. In addition, to obtain in-depth knowledge of genetic regulation of nutritional content compounds in fruits, tomato also represents an excellent model system for both basic and applied research for many reasons, including ease of growth in a wide range of environments, a short life-cycle, and well-developed genetic and genomic tools (Foolad 2007; Barone et al. 2008). Consequently, fruit quality in terms of both nutritional and organoleptic quality [flavour, antioxidant, degrees Brix $\left({ }^{\circ} \mathrm{Bx}\right)$ content, etc.] has become a major focus of most traditional and innovative tomato breeding approaches. Such traits usually exhibit quantitative variation controlled by several genes, and influenced by environmental conditions (Schauer et al. 2008). Molecular markers allow the dissection of such quantitative traits into discrete quantitative trait loci (QTL), which can be located on the tomato genetic map (Frary et al. 2005). Moreover, the development of molecular marker-assisted selection (MAS) has enabled the construction of a population of introgression lines (ILs) (Eshed and Zamir 1995), where each IL includes single marker-defined introgressed genomic regions from the wild green-fruited species Solanum pennellii into the genomic background of the cultivated $S$. lycopersicum inbred variety M82. Overall, the IL population provides complete coverage of the wild-species genome and allows the reservoir of wild genes to be investigated. In particular, S. pennellii introgression lines were shown to be powerful material for dissecting plant yield and fruit quality (Gur and Zamir 2004; Rousseaux et al. 2005; Stevens et al. 2007). In addition, this population has already been used for mapping candidate genes and QTL for carotenoids, fruit weight and composition in sugars and acids, antioxidant compounds, volatile aromas and various metabolites (Lippman et al. 2007).

In our laboratory, two $S$. pennellii ILs (IL7-3 and IL12-4) were identified for producing higher antioxidant and soluble solids fruit content in comparison with the S. lycopersicum variety M82, following threeyear screening under greenhouse conditions. In particular, one QTL for high AsA content was identified in the introgressed region 12-4 (Di Matteo et al. 2010a) and one QTL for high AsA, phenols and ${ }^{\circ} \mathrm{Bx}$ content in the introgressed region 7-3 (Di Matteo et al. 2010b). The aim of this study was to produce new genetic materials with combined high fruit antioxidant and ${ }^{\circ} \mathrm{Bx}$ content. For this purpose, we pyramided these QTL in the genetic background of the cultivated processing tomato M82 with the aid of introgressionspecific molecular markers by selecting markers suitable for distinguishing the wild genome of S. pennellii from the cultivated S. lycopersicum.

Seeds from IL12-4 (LA4102), IL 7-3 (LA4066), and their parental line M82 (LA3475) were kindly provided by The Tomato Genetics Resource Centre (TGRC) (http://tgrc.ucdavis.edu/). Crosses between these lines were made in order to assay the interaction among QTL carried by introgressions 7-3 and 12-4. In particular, to better understand the genetic architecture of each QTL, the two ILs were crossed to the recurrent parent M82 (ILH7-3 and ILH12-4) and between them (ILH7-3+12-4). Markers that allowed differentiation between the wild and cultivated alleles were selected by exploring the Sol Genomics Network database (www.solgenomics.net/search/markers). To this end, markers on chromosomes 7 and 12 were sought in the chromosomal regions extending from 39 to $64 \mathrm{cM}$ and from 74 to $120 \mathrm{cM}$ for IL7-3 and IL12-4, respectively. Eighteen markers tested for targeting 7-3 and 12-4 introgressions showed polymorphism between parental lines. In particular, for 17 markers tested, PCR fragments amplified from each parental genotype were sequenced and restriction enzymes that discriminated genotypes were selected. Only in one case (AT3g 17000) was the difference due to an insertion/ deletion and no sequencing of PCR fragments was required. In total, four and three markers for introgression regions 7-3 and 12-4 were chosen, respectively (Table 1). These mapped approximately at the top, middle and bottom of each region. These markers were used to validate hybrid genotypes as harbouring alleles from both parents.

The hybrids and their parental lines (five plants/ genotype) were then grown in a cold greenhouse according to a completely randomized design. Fruits were collected when $75 \%$ were full-sized and redripe, softening had increased and the inside of the columella was completely red. Chemical properties of 10-20 red-ripe fruit per genotype were assessed. A modified version of the enzymatic method described by Stevens et al. (2006) was used for reduced AsA 
Table 1 Selected markers for both introgression regions 7-3 and 12-4, which were used for screening ILH7-3, ILH12-4, ILH7$3+12-4$ and IL7-3+12-4

\begin{tabular}{|c|c|c|c|c|c|c|}
\hline \multirow{2}{*}{$\begin{array}{l}\text { Introgression } \\
\text { region }\end{array}$} & \multirow[t]{2}{*}{ Marker } & \multirow{2}{*}{$\begin{array}{l}\text { Position } \\
(\mathrm{cM})\end{array}$} & \multirow{2}{*}{$\begin{array}{l}\text { PCR product } \\
\text { (bp) }\end{array}$} & \multirow{2}{*}{$\begin{array}{l}\text { Restriction } \\
\text { enzyme }\end{array}$} & \multicolumn{2}{|c|}{ Restriction fragment size $^{\mathrm{a}}$ (bp) } \\
\hline & & & & & S. pennellii & S. lycopersicum \\
\hline $7-3$ & C2_At2g20830 & 43.00 & 550 & $B g l \mathrm{II}$ & $450+100$ & $380+100+70$ \\
\hline $7-3$ & C2_At1g17200 & 46.00 & 700 & HaeIII & $500+180$ & $500+200$ \\
\hline $7-3$ & C2_At1g53670 & 54.00 & 1,350 & $\operatorname{Taq} \mathrm{I}$ & $700+400$ & $1000+100$ \\
\hline $7-3$ & U220926 & 63.70 & 370 & AluI & $230+140$ & 370 \\
\hline $12-4$ & T0801 & 74.00 & 600 & $R s a \mathrm{I}$ & $340+180+60$ & $520+60$ \\
\hline $12-4$ & At4g31150 & 96.00 & 400 & EcoRV & 400 & $250+150$ \\
\hline $12-4$ & AT3g17000 & 115.00 & $1,300 / 1,100$ & - & & \\
\hline
\end{tabular}

cM distance refers to the Tomato-EXPEN 2000 map available at the Sol genomics network, http://solgenomics.net

a Only the digestion pattern that allowed distinction of $S$. pennellii from $S$. lycopersicum is reported

determination. Briefly, tomato fruits were ground in liquid nitrogen, and $500 \mathrm{mg}$ of powder was homogenized with $30 \mu \mathrm{L}$ of ice-cold $6 \%$ trichloroacetic acid (TCA). Samples were centrifuged for $20 \mathrm{~min}$ at $20,000 \mathrm{~g}$ at $4{ }^{\circ} \mathrm{C} .20 \mu \mathrm{L} 0.4 \mathrm{M}$ phosphate buffer and $10 \mu \mathrm{L} \mathrm{H}_{2} \mathrm{O}$ were added to a $20 \mu \mathrm{L}$ aliquot of the supernatant followed by the addition of $80 \mu \mathrm{L}$ of color reagent. The color reagent was made up as follows: solution $\mathrm{A}=31 \%$ orthophosphoric acid, $4.6 \%(\mathrm{w} / \mathrm{v})$ TCA and $0.6 \%$ (w/v) iron chloride $\left(\mathrm{FeCl}_{3}\right)$; solution $\mathrm{B}=4 \%$ 2,2-dipyridyl (w/v; made up in $70 \%$ ethanol). Solutions A and B were mixed 2.75 parts A to 1 part B. After incubation at $37{ }^{\circ} \mathrm{C}$ for $40 \mathrm{~min}$, the absorbance was read at $550 \mathrm{~nm}$ using a NanoPhotometer $^{\mathrm{TM}}$ (Implen).

Folin-Ciocalteu's procedure (Singleton and Rossi 1965) was used for phenol quantification, and a portable refractometer ATAGO Model ATC-1 for measuring soluble solids. The AsA concentration was expressed in $\mu \mathrm{mol} \mathrm{\textrm {g } ^ { - 1 }}$ fresh weight $(\mathrm{FW})$ according to the standard curve $\mathrm{A}_{525}=3.6593 \times \mu \mathrm{mol}$ AsA, designed over a dynamic range of $0-0.7 \mu$ mol AsA $\left(R^{2}=0.9982\right)$. Concentrations were then converted into $\mathrm{mg}(100 \mathrm{~g} \mathrm{FW})^{-1}$. Total phenolic concentration was expressed in terms of $\mathrm{mg}$ of gallic acid equivalents (GAEs) $\left(100 \mathrm{~g} \mathrm{FW}^{-1}\right.$, based on a gallic acid standard curve designed over a dynamic range of $0-125$, which is $\mathrm{A}_{760}=(0.0234 \times \mu \mathrm{g}$ gallic acid $)-0.0776\left(R^{2}=\right.$ 0.995). Soluble solids content was expressed on a ${ }^{\circ} \mathrm{Bx}$ scale. Data collected were analyzed by means of parametric tests by using SPSS (Statistical Package for Social Sciences) Package 6 version 15.0. Significance was determined by comparing mean values of ILs and
ILHs to the control M82 through a factorial analysis of variance (one-way ANOVA) with LSD post hoc test at a significance level of 0.05 .

The AsA fruit content of plants heterozygous for introgressions 7-3 (ILH7-3) and 12-4 (ILH12-4) was no different from the control, leading us to hypothesize the recessive inheritance of both QTL controlling AsA in the wild species $S$. pennellii (Fig. 1). By contrast, ILH7-3+12-4 plants displayed a significantly higher AsA content $(+39 \%)$ with respect to M82 control, thus suggesting a positive epistatic interaction between the AsA QTL mapping to the two introgressed regions (7-3 and 12-4). Looking at the total phenol content of the ILH7-3+12-4 fruits we can assume the recessive inheritance for the phenol QTL with no more speculation, since the data for ILH7-3 were missing because not enough fruit samples were available for the statistical analyses. Finally, ${ }^{\circ} \mathrm{Bx}$ contents of ILH7-3+12-4 and ILH7-3 were significantly higher $(+22 \%)$ than the control leading us to suppose the dominant inheritance of this trait.

In order to pyramid these QTL in new genetic materials, the QTL under study must be stabilized at the homozygous condition. For this purpose, the ILH7-3+12-4 genotypes were selfed to obtain F2 genotypes. Indeed, genotypes obtained by intercrossing the two ILs IL7-3 and IL12-4 are homozygous in all regions that carry the M82 genetic background, but heterozygous only in the two introgression regions. Therefore, by selfing ILH7-3+12-4 the segregation of both introgressed heterozygous regions will occur and $1 / 16$ of genotypes simultaneously homozygous in these two regions is expected. Sixty-eight F2 


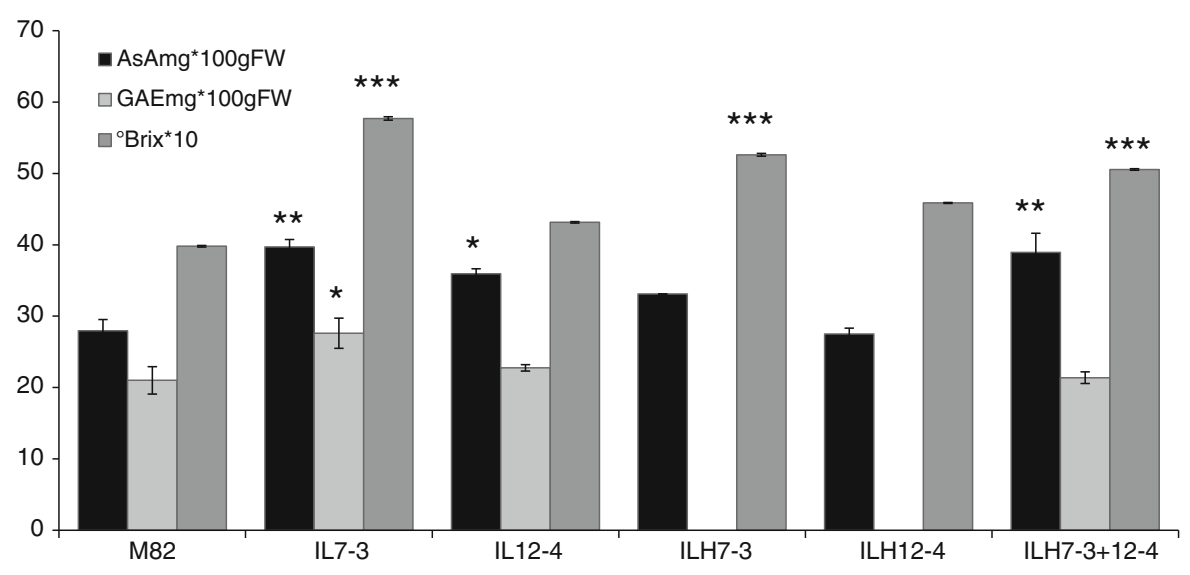

Fig. 1 Ascorbic acid, total phenols and ${ }^{\circ} \mathrm{Bx}$ content in red-ripe fruit from the tomato IL7-3, IL12-4, ILH7-3, ILH12-4, ILH7$3+12-4$ genotypes and the parental line M82. The mean values
$( \pm \mathrm{SE})$ are reported for at least three plant replicates grown under greenhouse conditions in 2009. Asterisks indicate statistically significant differences compared to M82 $(P<0.05)$

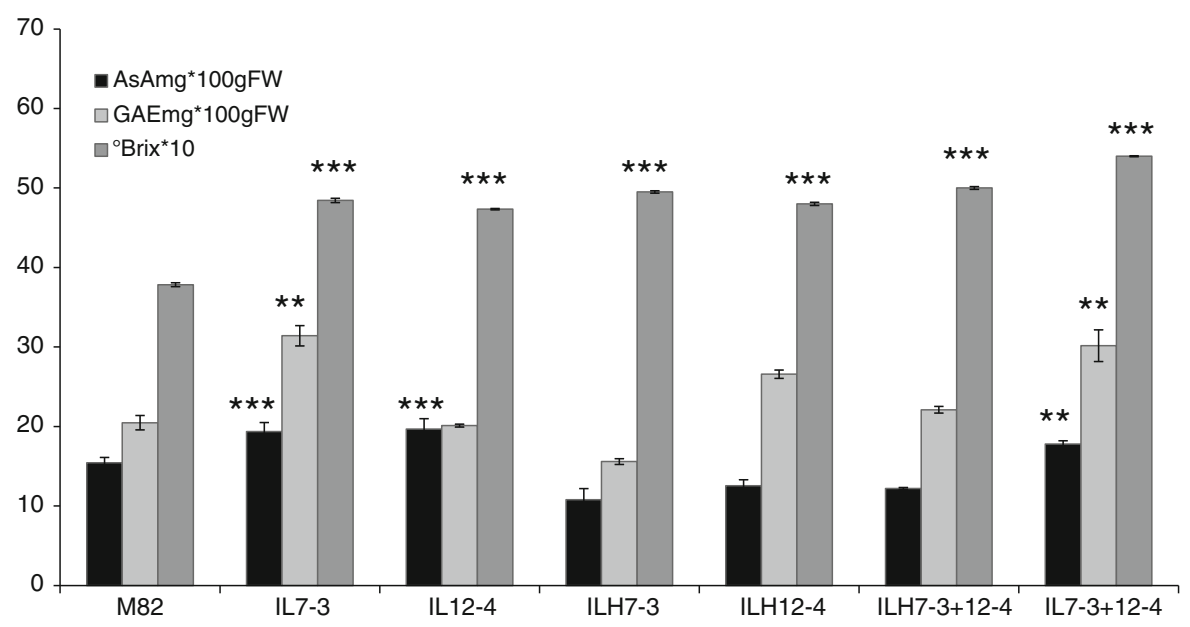

Fig. 2 Ascorbic acid, total phenols and ${ }^{\circ} \mathrm{Bx}$ content in red-ripe fruit from the tomato IL7-3, IL12-4, ILH7-3, ILH12-4, ILH7$3+12-4$, IL 7-3+12-4 genotypes and the parental line M82. The

genotypes obtained by selfing ILH7-3+12-4 were screened by the seven markers previously chosen, which allowed selection of four genotypes carrying both introgressions 7-3 and 12-4 at the homozygous condition (IL7-3+12-4). This number is consistent with the expected value of 4.25 genotypes out of a total of 68 analyzed. The double homozygous plants obtained, which were stable in all their genomic regions, were then selfed and F3 families (10 plants/ family in three replicates) were field-grown in southern Italy.

As shown in Fig. 2, the IL7-3+12-4 displayed significantly higher AsA, total phenol and ${ }^{\circ} \mathrm{Bx}$ content mean values $( \pm \mathrm{SE})$ are reported for at least seven replicates of plants field-grown in 2010. Asterisks indicate statistically significant differences compared to M82 $(P<0.05)$

with respect to M82. The AsA content of the two ILH7-3 and ILH12-4 was confirmed to be no different from M82 under field conditions also, thus strengthening the recessive inheritance of the AsA QTL. As for AsA QTL, our hypothesis on the inheritance mode of the other two QTL for phenol and ${ }^{\circ} \mathrm{Bx}$ was supported by the results obtained in the field. The ILHs (ILH7-3, ILH12-4 and ILH7-3+12-4) showed a similar phenol content to the control and a significantly higher ${ }^{\circ} \mathrm{Bx}$ value than $\mathrm{M} 82$, confirming the recessive and dominant inheritance of phenols and ${ }^{\circ} \mathrm{Bx}$ QTL, respectively. Comprehensively, despite the values of the different metabolites measured depending on the 


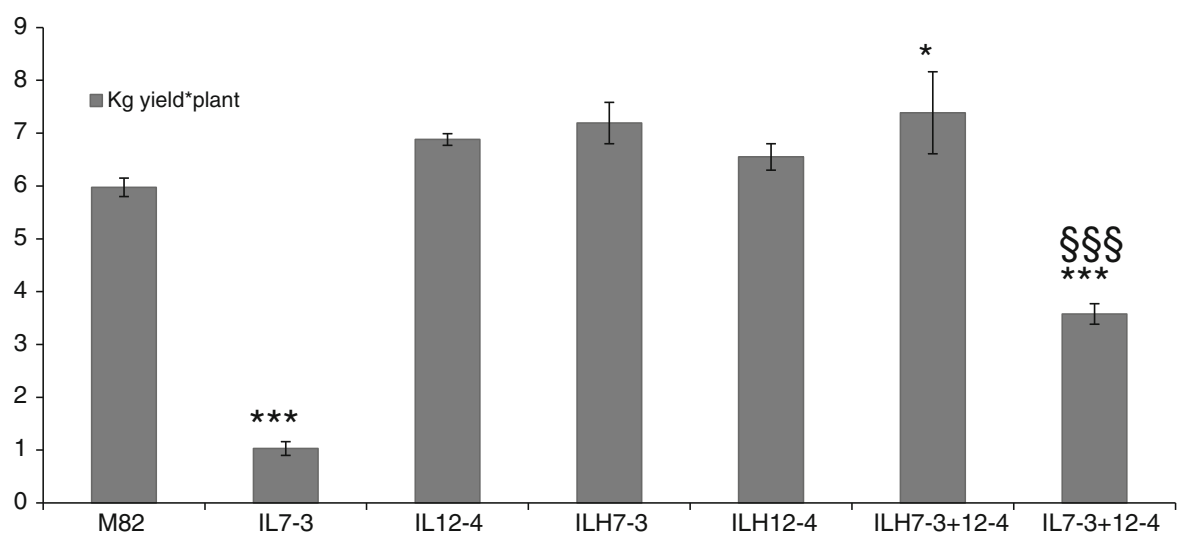

Fig. 3 Total plant yield (kg/plant) of IL7-3, IL12-4, ILH7-3, ILH12-4, ILH7-3+12-4, IL7-3+12-4 genotypes and the parental line M82. The mean values $( \pm \mathrm{SE})$ are reported for at least seven plant replicates grown in the open field in 2010. Asterisks indicate statistically significant differences compared to M82 $(P<0.05)$, section symbol indicates statistically significant differences compared to IL7-3 $(P<0.05)$

genetic material for a breeding scheme aiming to increase antioxidant content in tomato fruit. All these genetic materials could be of extreme interest to seed companies and the plant breeding industry, which could use them as a starting point to generate new hybrids or improved varieties.

Indeed, IL heterosis has already progressed beyond scientific publications into practical use in agriculture. A QTL pyramiding study with three independent yield-promoting introgressions resulted in a hybrid with $50 \%$ higher yields than leading commercial varieties in multiple environments and irrigation regimes (Gur et al. 2011). Moreover, introgressions originating from $S$. pennellii were introduced into lines of processing tomato, and the resulting hybrid $\mathrm{AB} 2$ is presently a leading variety in California (http://www. ptab.org/ranking9.htm), which is the largest producer of processing tomatoes in the world. These real-world applications illustrate how 'exotic' alleles from interspecific diversity can enrich the genetic basis of cultivated plants to improve productivity (Lippman et al. 2007). In our study, the IL7-3+12-4 showed increased AsA $(+15 \%)$, phenol content $(+47 \%)$, and ${ }^{\circ} \mathrm{Bx}(+43 \%)$ but decreased yield $(-40 \%)$ with respect to M82. These results confirmed that crosses between ILs give a chance of obtaining useful material for breeding, even though in our case a better definition of the "useful" introgression region 7-3 is required, as also suggested for rice by Ashikari and Matsuoka (2006). This could be achieved by chromosome recombination between the IL7-3+12-4 decrease compared to IL7-3, thus obtaining suitable 
progeny and the recurrent parent followed by MAS that might lead to obtaining different sub-lines of the region carrying desirable and undesirable traits. Indeed, the information deriving from the complete sequencing of the tomato genome (Tomato Genome Consortium 2012) will shortly facilitate identification and mapping of candidate genes for antioxidant fruit content and plant production in the introgression region 7-3. Therefore, further investigation will facilitate the dissection of the introgressed region into reduced sub-lines, helping select those that decouple positive from negative traits.

Acknowledgments The authors thank Mark Walters for editing the manuscript. This work was funded by the MiPAF AGRONANOTECH and MiUR GENOPOM programs. Contribution no 269 from the DISSPAPA.

Open Access This article is distributed under the terms of the Creative Commons Attribution License which permits any use, distribution, and reproduction in any medium, provided the original author(s) and the source are credited.

\section{References}

Ashikari M, Matsuoka M (2006) Identification, isolation and pyramiding of quantitative trait loci for rice breeding. Trends Plant Sci 11(7):344-350

Barone A, Chiusano ML, Ercolano MR, Giuliano G, Grandillo S, Frusciante L (2008) Structural and functional genomics of tomato. Int J Plant Genomics 2008:820274. doi: $10.1155 / 2008 / 820274$

Causse M, Friguet C, Coiret C, Lépicier M, Navez B, Lee M, Holthuysen N, Sinesio F, Moneta E, Grandillo S (2010) Consumer preferences for fresh tomato at the European scale: a common segmentation on taste and firmness. J Food Sci 75(9):S531-S541

Demmig-Adams B, Adams WW III (2002) Antioxidants in photosynthesis nutrition. Science 298:2149-2153

Di Matteo A, Sacco A, Anacleria A, Pezzotti M, Delledonne M, Ferrarini A, Frusciante L, Barone A (2010a) The ascorbic acid content of tomato fruits is associated with the expression of genes involved in pectin degradation. BMC Plant Biol 10:163-173

Di Matteo A, Sacco A, Ruggieri V, Trotta N, Nunziata A, Barone A (2010b) Transcriptional network controlling antioxidants in tomato fruit. J Biotechnol 150S:111-112

Eshed Y, Zamir D (1995) An introgression line population of Lycopersicon pennellii in the cultivated tomato enables the identification and fine mapping of yield-associated QTLs. Genetics 141:1147-1162

Foolad MR (2007) Genome mapping and molecular breeding of tomato. Int J Plant Genomics 2007:64358. doi:10.1155/ 2007/64358

Frary A, Xu Y, Liu J, Mitchell S, Tedeschi E, Tanksley SD (2005) Development of a set of PCR-based anchor markers encompassing the tomato genome and evaluation of their usefulness for genetics and breeding experiments. Theor Appl Genet 111:291-312

Gur A, Zamir D (2004) Unused natural variation can lift yield barriers in plant breeding. PLoS Biol 2(10):e245. doi:10.1371/journal.pbio.0020245

Gur A, Semel Y, Osorio S, Friedmann M, Seekh S, Mohammad BGA, Pleban T, Gera G, Fernie AR, Zamir D (2011) Yield quantitative trait loci from wild tomato are predominately expressed by the shoot. Theor Appl Genet 122:405-420

Lippman ZB, Semel Y, Zamir D (2007) An integrated view of quantitative trait variation using tomato interspecific introgression lines. Curr Opin Genet Dev 17:542-545

Rousseaux MC, Jones CM, Adams D (2005) QTL analysis of fruit antioxidants in tomato using Lycopersicon pennellii introgression lines. Theor Appl Genet 111:1396-1408

Schauer N, Semel Y, Balbo I, Steinfath M, Repsilber D, Selbig J, Pleban T, Zamir D, Fernie AR (2008) Mode of inheritance of primary metabolic traits in tomato. Plant Cell 20:509-523

Singleton VL, Rossi JA (1965) Colorimetry of total phenolics with phosphomolybdic-phosphotungstic acid reagents. Am J Enol Viticult 16:144-158

Stevens R, Buret M, Garchery CC, Carretero Y, Causse M (2006) Technique for rapid small-scale analysis of vitamin $\mathrm{C}$ levels in fruit and application to a tomato mutant collection. J Agric Food Chem 54:6159-6165

Stevens R, Buret M, Duffe' P, Garchery C, Baldet P, Rothan C, Causse M (2007) Candidate genes and quantitative trait loci affecting fruit ascorbic acid content in three tomato populations. Plant Physiol 143:1943-1953

Tomato Genome Consortium (2012) The tomato genome sequence provides insights into fleshy fruit evolution. Nature 485(7400):635-641. doi:10.1038/nature11119 